\title{
Mental healthcare for psychiatric inpatients during the COVID-19 epidemic
}

Shen $\mathrm{Li}^{1}{ }^{1}$ Yong Zhang ${ }^{2}$

\begin{abstract}
During this 2019 coronavirus disease (COVID-19) pneumonia epidemic, some experts have expressed concern for the mental healthcare of different types of population groups. However, hospitalised patients with severe mental illness are seemingly overlooked. Psychiatric patients are still a vulnerable group who need to obtain more attention and respect, particularly during the COVID-19 outbreak in China. In this commentary, we briefly introduce the situation of hospitalized patients with severe mental illness and suggest some effective measures that should be rapidly undertaken to reverse current challenges.
\end{abstract}

During the surge in 2019 coronavirus disease (COVID-19) transmissions, patients with severe mental disorders were also inevitably affected. As of 20 February 2020, 323 patients with severe mental disorders have been confirmed to have COVID-19 pneumonia, with an additional 43 suspected cases.

Since January 2020, the National Health Commission of China has published several guidelines for psychological crisis intervention and initiated psychological assistance hot lines for the COVID-19. ${ }^{1}$ On 18 February 2020, the Chinese Medical Association's Psychiatry branch issued a consensus on the diagnosis and treatment procedures for mental disorders during the prevention and control of major emergent infectious diseases (COVID-19), ${ }^{2}$ which addressed treatment and safety for individuals with severe mental disorders. However, the mental healthcare for this population has not been sufficiently paid attention to during the crisis.

In China, mental disorders have significantly increased across China

\footnotetext{
${ }^{1}$ Department of Psychiatry, College of Basic Medical Sciences, Tianjin Medical University, Tianjin, China ${ }^{2}$ Department of Bipolar Disorder, Tianjin Anding Hospital, Tianjin, China

Correspondence to Dr Yong Zhang;

zhangyong@tjmhc.com
}

in the past 30 years. The latest nationwide survey reported the prevalence of most mental disorders in an entire lifetime was $16.6 \%{ }^{3}$ indicating that a large proportion of the population has suffered from mental disorders. Unfortunately, most psychiatric patients had to be isolated in the hospital during the COVID-19 outbreak, which exacerbated their psychological distress.

In this COVID-19 pneumonia epidemic, some experts have expressed concern for the mental healthcare of medical workers, ${ }^{4}$ older adults, ${ }^{5}$ confirmed and suspected patients as well as other people in quarantine. ${ }^{6}$ Further, according to the national commission initiate, emergency guidelines were formulated for these different groups. However, hospitalised patients with severe mental illness were seemingly overlooked. Compared with patients from other departments, psychiatric patients encountered more barriers and problems. For instance, psychiatric patients had to stay in closed wards, their family visiting was cancelled due to fear of transmission of the novel coronavirus, smartphones and other electronic equipment were not permitted in the ward and no online information was made available. Meanwhile, psychiatric patients are more susceptible to COVID-19 contamination in relatively isolated settings. It was reported that 50 psychiatric patients and 30 medical staff suffered from COVID-19 at the Wuhan Mental Health Centre; recently, a similar tragedy occurred in a psychiatric hospital of South Korea. Unluckily, some small to medium sized psychiatric hospitals also refused to receive new inpatients because of poor medical conditions, which possibly deteriorated psychotic symptoms for patients with mental illness. At present, a great number of psychiatrists and clinical psychologists are flooding into Wuhan to support the front line.
However, the psychological needs of hospitalised patients with mental illness are not likely to be fulfilled.

Accordingly, some effective measures should be rapidly undertaken to reverse these challenges. First, current policy and regulation issued by the government ought to highlight the needs of psychiatric patients and their families or supplement-related rules to provide mental health services. Second, the provision for communication between psychiatric inpatients and their families should be considered by hospitals. Some electronic devices such as smartphones should be fixed in the wards and managed by nurses, so both patients and family members have a channel to alleviate the stress and negative emotions caused by isolation and loneliness. Third, in the published guidance for mental healthcare that has been initiated in China, ${ }^{1}$ we suggest that the combination of online psychological intervention and face-to-face counselling should be widely adopted in psychiatric hospitals nationwide. Finally, the teams responsible for patient's psychological support and treatment should include not only psychiatrists and psychologists but also psychiatric nurses, social workers, volunteers and family members, in order to unify patients within a supportive system in which every member is able to smoothly exchange information and seek better solutions for mental issues.

Despite Chinese guidelines for psychological crisis interventions and expert consensus for the treatment of psychiatric patients during the COVID-19 epidemic, psychiatric patients are still a vulnerable group who need more attention and respect, particularly during the COVID-19 outbreak in China. Healthcare workers and authorities should jointly deal with mental health problems to offer effective and timely psychological services for psychiatric patients.

Contributors Study design: YZ; writing: YZ and SL.

Funding The authors have not declared a specific grant for this research from any funding agency in the public, commercial or not-for-profit sectors.

Competing interests None declared.

Patient consent for publication Not required. 
Provenance and peer review Not commissioned; externally peer reviewed.

\section{(2) \\ OPEN ACCESS}

Open access This is an open access article distributed in accordance with the Creative Commons Attribution Non Commercial (CC BY-NC 4.0) license, which permits others to distribute, remix, adapt, build upon this work non-commercially, and license their derivative works on different terms, provided the original work is properly cited, appropriate credit is given, any changes made indicated, and the use is non-commercial. See: http://creativecommons.org/licenses/by-nc/4.0/.

(C) Author(s) (or their employer(s)) 2020. Re-use permitted under CC BY-NC. No commercial re-use. See rights and permissions. Published by BMJ.
Check for updates

To cite Li S, Zhang Y. General Psychiatry Epub ahead of print: [please include Day Month Year]. doi:10.1136/ gpsych-2020-100216

Received 3 March 2020

Revised 16 March 2020

Accepted 24 March 2020

General Psychiatry 2020;33:e100216.

doi:10.1136/gpsych-2020-100216

\section{REFERENCES}

1 Liu S, Yang L, Zhang C, et al. Online mental health services in China during the COVID-19 outbreak. Lancet Psychiatry 2020;7:e17-18.

2 Psychiatry CMAo. Diagnosis and treatment process and path of mental disorders during the prevention and control of major emergent infectious diseases (2019 coronavirus disease). Chinese Journal of Psychiatry 2020;53:E002

3 Huang $\mathrm{Y}$, Wang $\mathrm{Y}$, Wang $\mathrm{H}$, et al. Prevalence of mental disorders in China: a crosssectional epidemiological study. Lancet Psychiatry 2019;6:211-24.

4 Chen Q, Liang M, Li Y, et al. Mental health care for medical staff in China during the COVID-19 outbreak. Lancet Psychiatry 2020; 7:e15-16.

5 Yang Y, Li W, Zhang Q, et al. Mental health services for older adults in China during the COVID-19 outbreak. Lancet Psychiatry 2020;7:e19.

6 Duan L, Zhu G. Psychological interventions for people affected by the COVID-19 epidemic. Lancet Psychiatry 2020;7:300-2.

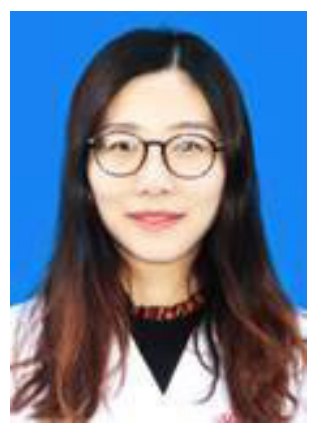

Shen Li completed a bachelor's degree in 2008, a Master's degree in 2011, and a Ph.D in Psychiatry from Tianjin Medical University, Tianjin, China in 2017. Since 2011, she has been a teacher and psychiatrist at the Department of Psychiatry, Tianjin Medical University, Tianjin, China. She is now accepting postdoctoral training in Mclean Hospital, a Harvard Medical School affiliate. Her research interests include molecular biology on the response of antipsychotics, and multimodal approaches to stratify patients who experience their first episode psychosis into homogeneous subgroups. 